\title{
Ultrasound for Critical Care Physicians: Caught in the Act
}

\author{
Uzoamaka Ogbonnah MD' \\ Isaac Tawil MD2 \\ Trenton C. Wray MD \\ Michel Boivin MD' \\ ${ }^{1}$ Department of Internal Medicine \\ ${ }^{2}$ Department of Emergency Medicine \\ University of New Mexico School of Medicine \\ Albuquerque, NM USA
}

A 16-year-old man was brought to the Emergency Department via ambulance after a fall from significant height. On arrival to the trauma bay, the patient was found to be comatose and hypotensive with a blood pressure of $72 / 41 \mathrm{~mm} / \mathrm{Hg}$. He was immediately intubated, started on norepinephrine drip with intermittent dosing of phenylephrine, and transfused with 3 units of packed red blood cells. He was subsequently found to have extensive fractures involving the skull and vertebrae at cervical and thoracic levels, multi-compartmental intracranial hemorrhages and dissection of the right cervical internal carotid and vertebral arteries. He was transferred to the intensive care unit for further management of hypoxic respiratory failure, neurogenic shock and severe traumatic brain injury. Following admission, the patient continued to deteriorate and was ultimately declared brain dead 3 days later. The patient's family opted to make him an organ donor

On ICU day 4, one day after declaration of brain death, while awaiting organ procurement, the patient suddenly developed sudden onset of hypoxemia and hypotension while being ventilated. The patient had a previous trans-esophageal echo (TEE) the day prior (Video 1). A repeat bedside TEE was performed revealing the following image (Video 2).

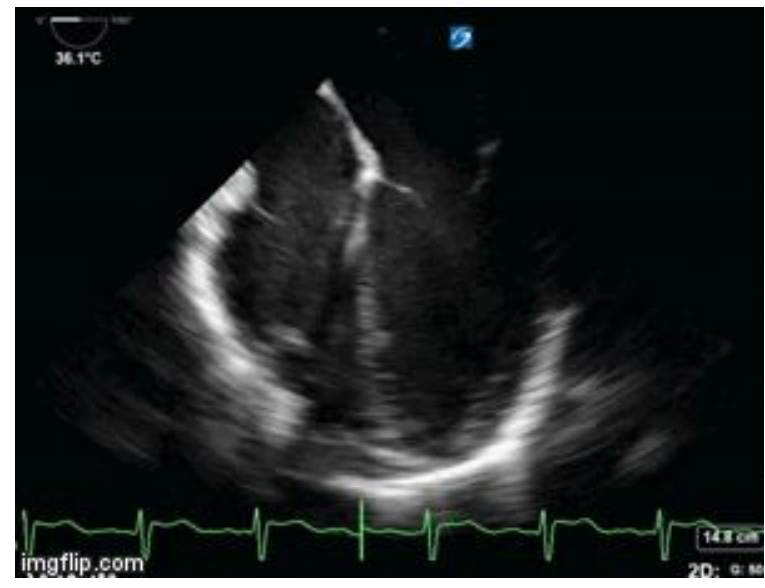

Video 1. Mid-esophageal four chamber view of the right and left ventricle PRIOR to onset of hypoxemia. 


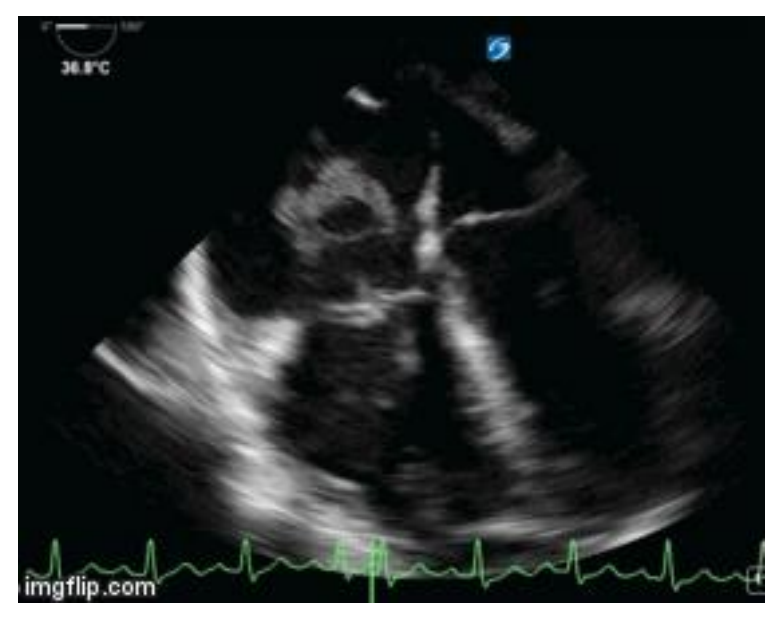

Video 2. Mid-esophageal four chamber view of the right and left ventricle AFTER deterioration.

What is the cause of the patient's sudden respiratory deterioration?

1. Atrial Myxoma

2. Fat emboli syndrome

3. Thrombus in-transit and pulmonary emboli

4. Tricuspid valve endocarditis 


\section{Correct! \\ 3. Thrombus in-transit and pulmonary emboli}

Multiple thrombi can be seen in transit through the right atrium. A second thrombus is seen near the subvalvular apparatus. Multiple other small thrombi are also visualized. Aside from obvious clot caught in transit, the ultrasound displays other classical signs that can be associated with pulmonary embolism. When compared to the TEE performed 2 days prior (video 1), the right ventricle was now noted to be moderately to severely dilated with an $\mathrm{RV} / \mathrm{LV}$ ratio of approximately $1.2 / 1$ (1). The right ventricular function was also now moderately reduced with hypokinesis of the base and relative sparing of the apex (McConnell's sign) (2). This sign is associated with acute pulmonary embolism and also acute right ventricular infarction. The image below shows video 2 , with an "M" placed just below the point where the sparing of the apical motion meets the hypokinesis of the free wall (hinge point) (video 3). Also noted is a bowing intra-atrial septum (right to left) indicated elevated right atrial pressures.

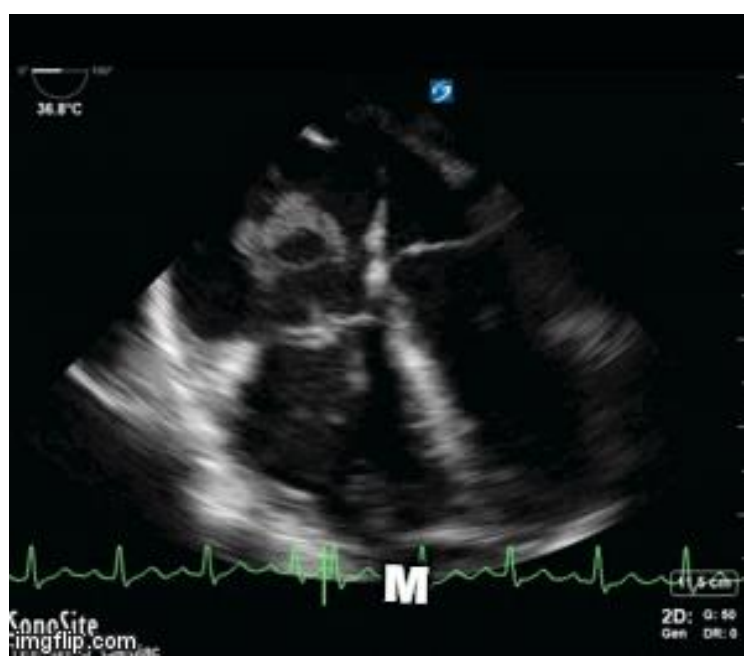

Video 3. Mid-esophageal four chamber view of the right and left ventricle AFTER deterioration, with letter " $\mathrm{M}$ " placed just below the "hinge point" associated with McConnell's sign.

The source of the emboli was determined to be thrombus adherent other patient's intravascular cooling catheter, used to normalize temperature in this patient with traumatic brain injury. Vena caval thrombus is a described complication of these types of catheters.

\section{References}

1. Konstantinides SV, Torbicki A, Agnelli G, Danchin N, Fitzmaurice D, Galiè N, et al. 2014 ESC guidelines on the diagnosis and management of acute pulmonary embolism. Eur Heart J. 2014;35:3033-69. [CrossRef] [PubMed]

2. Lodato JA, Ward RP, Lang RM. Echocardiographic predictors of pulmonary embolism in patients referred for helical CT. Echocardiography. 2008;25:584-90. [CrossRef] [PubMed] 